\title{
Childhood underweight, weight gain during childhood to adolescence/young adulthood and incidence of adult metabolic syndrome in the SUN (Seguimiento Universidad de Navarra) Project
}

\author{
Adriano M Pimenta ${ }^{1}$, Juan-José Beunza ${ }^{2}$, Almudena Sanchez-Villegas $^{3}$, \\ Maira Bes-Rastrollo ${ }^{2}$ and Miguel A Martinez-Gonzalez ${ }^{2, *}$ \\ 'Department of Maternal and Child Nursing and Public Health, Nursing School, Federal University of Minas \\ Gerais, Belo Horizonte, MG, Brazil: ${ }^{2}$ Department of Preventive Medicine and Public Health, Medical School \& \\ Clinic, University of Navarra, Irunlarrea 1, CP 31080, Pamplona, Navarra, Spain: ${ }^{3}$ Department of Clinical \\ Sciences, University of Las Palmas de Gran Canaria, Las Palmas de Gran Canaria, Spain
}

Submitted 1 May 2010: Accepted 22 September 2010: First published online 17 November 2010

\begin{abstract}
Objective: To assess associations between childhood body weight, weight gain during childhood to adolescence/young adulthood and incidence of adult metabolic syndrome (MetS).

Design: A dynamic prospective cohort study (the SUN Project; Seguimiento Universidad de Navarra). Participants were asked to select which of nine body images most closely represented their body shape at ages 5 and 20 years, and it was used as a proxy of BMI. An incident case of MetS was diagnosed according to criteria of the International Diabetes Federation. Associations between childhood body weight, weight gain during childhood to adolescence/young adulthood and incidence of adult MetS were estimated by multiple-adjusted odds ratios and their 95\% confidence intervals.

Setting: University of Navarra, Spain.

Subjects: The study included 5317 university graduates, followed-up for a median of $6 \cdot 1$ years.

Results: The incidence of MetS was $2 \cdot 9 \%$ ( $1.7 \%$ in women and $5 \cdot 1 \%$ in men). Among men, body shape at age 5 years was inversely related to adult MetS $(\mathrm{OR}=0 \cdot 83,95 \%$ CI $0 \cdot 72,0 \cdot 97$ ), whereas weight gain during childhood to adolescence/young adulthood was directly associated with adult MetS (OR $=1 \cdot 49$, $95 \%$ CI $1 \cdot 01,2 \cdot 18)$; both childhood underweight (OR $=5 \cdot 20,95 \%$ CI $1 \cdot 87,14 \cdot 50)$ and childhood obesity $(\mathrm{OR}=4 \cdot 66,95 \% \mathrm{CI} 1 \cdot 40,15 \cdot 51)$ increased the likelihood of adult MetS. No association was apparent among women.

Conclusions: These results support treating childhood underweight and weight gain during childhood to adolescence/young adulthood as part of comprehensive adult MetS prevention efforts in men.
\end{abstract}

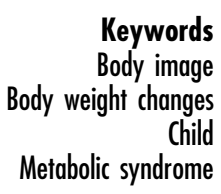

Keywords

Body image

child

Metabolic syndrome
Metabolic syndrome (MetS) is characterized by the clustering of cardiovascular risk factors such as obesity, dyslipidaemia, insulin resistance, hyperinsulinaemia, glucose intolerance and hypertension ${ }^{(1)}$. This syndrome is recognized as an important public health concern worldwide, owing to its prevalence of over $20 \%$ in adult populations around the world ${ }^{(1-4)}$ and also to its strong association with CVD and type 2 diabetes, both main causes of death on a global scale ${ }^{(1,5,6)}$.

In general, there are differences in the prevalence of MetS and its components among men and women. MetS has been more frequent in men than in women, but the prevalence has risen particularly in young women, where it is mainly driven by obesity. Furthermore, the presence of impaired glucose tolerance is higher among women, whereas the other MetS components are more common among men until women enter the menopause period ${ }^{(7)}$.

Some studies have also consistently shown high rates of MetS in people who were born small, because insulin resistance, which is recognized as a key element of this syndrome ${ }^{(8)}$, could be a genetically 'programmed' response to fetal undernutrition ${ }^{(9)}$. However, this hypothesis has been criticized for the reason that the prevalence of underweight newborn children is decreasing whereas the 
frequency of MetS is increasing in Western countries. Thus, it has been suggested that the origin of adulthood risk of this syndrome and other chronic diseases could be associated with somatic growth during childhood, and not necessarily to intra-uterine growth ${ }^{(10)}$. The weight change at different periods of early life may have differential effects on the acquisition of fat or lean adult mass and on the development of chronic disorders ${ }^{(11)}$.

Therefore, the aim of the present study was to assess the associations between childhood body weight, weight gain during childhood to adolescence/young adulthood and incidence of adult MetS among participants in the SUN (Seguimiento Universidad de Navarra, University of Navarra Follow-up) Project, a dynamic cohort study.

\section{Methods}

\section{Study population}

The SUN Project is a multi-purpose, dynamic Spanish cohort based on former students of the University of Navarra, registered professionals from some Spanish provinces and university graduates from other associations ${ }^{(12)}$. Information on exposure and outcome is gathered by mailed questionnaires collected biennially. Persons who do not reply to the follow-up questionnaires are sent up to five additional mailings.

The baseline questionnaire included 554 questions about a wide array of characteristics: sociodemographic (e.g. sex, age, marital status), anthropometric (e.g. weight, height), body shape at ages 5, 20, 30 and 40 years (e.g. nine-level body image scheme in each age), lifestyle and health-related habits (e.g. smoking status, physical activity), diet (e.g. food consumption, alcohol intake), obstetric history for women (e.g. pregnancy) and medical history (e.g. prevalence of diseases - including hypertension, diabetes, obesity, dyslipidaemia, cancer, myocardial infarction, stroke and atrial fibrillation - and medication use). The 2-year, 4-year, 6-year and 8-year follow-up questionnaires queried changes in some characteristics presented above (weight, lifestyle and health-related habits, diet, incidence of disease, pregnancy). The last two questionnaires also gathered additional information like self-reported waist circumference $(\mathrm{cm})$, blood pressure (mmHg), TAG (mg/dl), HDL cholesterol (HDL-C; mg/dl) and plasma glucose $(\mathrm{mg} / \mathrm{dl})$.

The recruitment of participants started in December 1999 and is permanently ongoing because it is a dynamic cohort. The overall follow-up rate approaches $90 \%$. Before June 2006, 15786 participants had answered the baseline questionnaire. From them, we excluded participants lost to follow-up ( $n$ 1449). Because the criteria to diagnose an incident case of MetS were collected only in the 6-year and 8-year follow-up questionnaires, persons who did not respond to these questionnaires were excluded, even though they answered other follow-up questionnaires ( $n$ 3621). Additionally, we also excluded participants with missing values for body weight variables ( $n$ 406); those who had chronic diseases (hypertension, diabetes, obesity and dyslipidaemia) at baseline, 2-year and 4-year follow-up ( $n$ 4697); those who reported extremely low $(<3347 \mathrm{~kJ} / \mathrm{d}$ for men, $<2092 \mathrm{~kJ} / \mathrm{d}$ for women) or high ( $>16736 \mathrm{~kJ} / \mathrm{d}$ for men, $>14644 \mathrm{~kJ} / \mathrm{d}$ for women) values for total energy intake, because they were more likely to have failed to properly complete the questionnaire ( $n$ 254); and pregnant women ( $n$ 42). Some participants met more than one of these exclusion criteria. Finally, 5317 participants were included in analyses (median follow-up $=6 \cdot 1$ years).

The study was approved by the Institutional Review Board of the University of Navarra. Voluntary completion of the first questionnaire was considered to imply informed consent.

\section{Childhood body weight, weight change during childbood to adolescence/young adulthood and baseline body weight assessment}

Body shape was ascertained at the baseline questionnaire with a nine-level body image scheme (Fig. 1) which was validated by Stunkard and colleagues: body images F1/M1 to F3/M3 correspond to underweight $\left(\mathrm{BMI}<19.99 \mathrm{~kg} / \mathrm{m}^{2}\right)$; body images $\mathrm{F} 4 / \mathrm{M} 4$ and $\mathrm{F} 5 / \mathrm{M} 5$ correspond to normal weight $\left(\mathrm{BMI}=20 \cdot 00-24 \cdot 99 \mathrm{~kg} / \mathrm{m}^{2}\right)$; body images $\mathrm{F} 6 / \mathrm{M} 6$ and $\mathrm{F} 7 / \mathrm{M} 7$ correspond to overweight $\left(\mathrm{BMI}=25 \cdot 00-29 \cdot 99 \mathrm{~kg} / \mathrm{m}^{2}\right)$; and body images F8/M8 and F9/M9 correspond to obesity (BMI $\left.\geq 30 \cdot 00 \mathrm{~kg} / \mathrm{m}^{2}\right)^{(13)}$.

Participants were asked to select which of the nine body images most closely represented their body shape at ages 5 and 20 years. The validity of the remote recall of body shape was assessed among 181 participants from the Third Harvard Growth Study. Pearson correlations between recalled body shape and BMI were 0.60 for age 5 years and 0.66 for age 20 years $^{(14)}$.

The weight gain during childhood to adolescence/ young adulthood was estimated by the difference between body shapes at ages 5 and 20 years.

Participants' self-reported weight and height at the baseline questionnaire in the SUN Project were used to calculate BMI. This information was validated in a specific study; although correlation does not necessarily mean good agreement, BMI calculated with self-reported weight and height exhibited a high correlation with BMI calculated from measured weight and height (mean relative error $=1 \cdot 4 \%)^{(15)}$.

\section{Metabolic syndrome assessment}

MetS was defined according to the International Diabetes Federation (IDF) criteria $^{(16)}$. In the 6-year and 8-year follow-up questionnaires, self-reported information about these criteria was collected (waist circumference $(\mathrm{cm})$, blood pressure $(\mathrm{mmHg})$, TAG $(\mathrm{mg} / \mathrm{dl})$, HDL-C $(\mathrm{mg} / \mathrm{dl})$ and plasma glucose $(\mathrm{mg} / \mathrm{dl}))$. Waist circumference was 

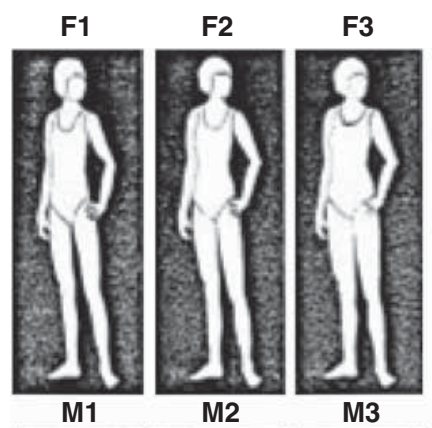

M3
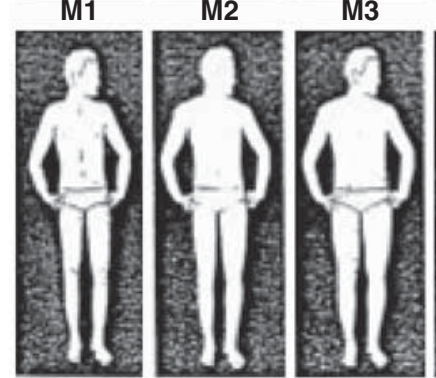

F4

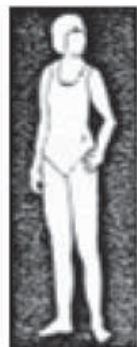

M4

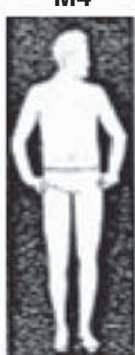

F5

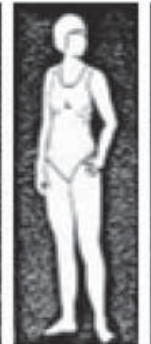

M5

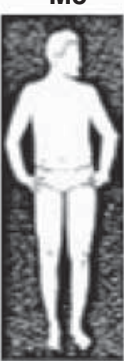

F6

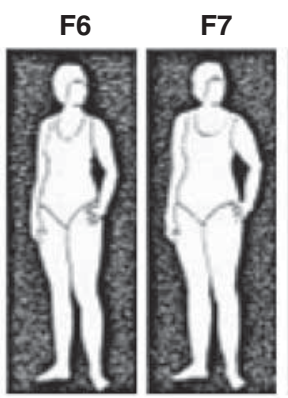

M6

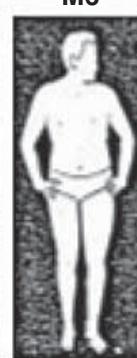

M7
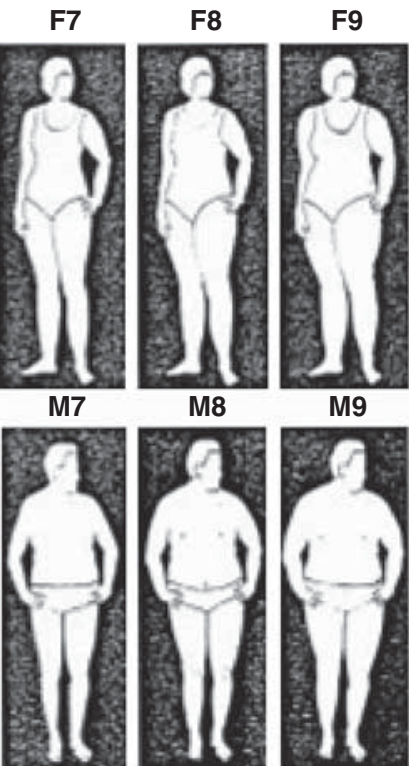

M9

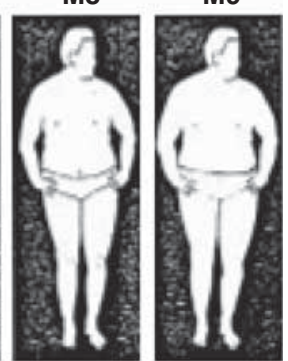

Fig. 1 Options presented to select body shape at age 5 and 20 years, according to sex. $F=$ female; $M=$ male; F1/M1 to F3/M3, underweight $\left(\mathrm{BMI}<19.99 \mathrm{~kg} / \mathrm{m}^{2}\right) ; \mathrm{F} 4 / \mathrm{M} 4$ and F5/M5, normal weight $\left(\mathrm{BMI}=20 \cdot 00-24 \cdot 99 \mathrm{~kg} / \mathrm{m}^{2}\right)$; F6/M6 to F9/M9, overweight or obese $\left(\mathrm{BMl} \geq 25 \cdot 00 \mathrm{~kg} / \mathrm{m}^{2}\right)$

measured in a horizontal plane, midway between the inferior margin of the ribs and the superior border of the iliac crest $^{(17)}$. All participants were sent a tape measure with the 6-year and 8-year follow-up questionnaires, and an explanation of how to measure their waist. If BMI was $\geq 30 \cdot 00 \mathrm{~kg} / \mathrm{m}^{2}$, central obesity was assumed and waist circumference not taken into account ${ }^{(16)}$.

The validities of the self-reported MetS components were assessed in a specific study with 287 participants in the SUN Project. Significant Pearson correlation coefficients $(P<0 \cdot 001)$, range $0 \cdot 5$ to $0 \cdot 9$, between self-reported MetS components and their direct assessments by an experienced physician were demonstrated ${ }^{(18)}$.

\section{Statistical methods}

The differences between the participants with respect to some characteristics were estimated with ANOVA or Pearson's $\chi^{2}$ test.

Three non-conditional logistic regression models were fit. Two of them were fitted to assess the relationship between childhood body weight and incidence of adult MetS: (i) in the first model, OR and their 95\% CI were calculated considering the normal category of BMI at age 5 years (body images F4/M4 and F5/M5, corresponding to $\mathrm{BMI}=20 \cdot 00-24 \cdot 99 \mathrm{~kg} / \mathrm{m}^{2}$ ) as the reference; (ii) in the second model, OR and their 95\% CI were calculated considering the body shape at age 5 years as a continuous variable. The third model was fitted to assess the relationship between weight gain during adolescence/young adulthood and incidence of adult MetS: (iii) in this model, OR and their 95\% CI were calculated considering the weight gain from 5 to 20 years as a continuous variable. To avoid reverse causality, participants with age less than or equal to 30 years at baseline ( $n$ 3273) were excluded from these analyses. Potential confounders included in all models were age and BMI at baseline.

All analyses were stratified by sex. All $P$ values presented are two-tailed; $P<0.05$ was considered statistically significant. The SPSS statistical software package for Windows version $15 \cdot 0$ (SPSS Inc., Chicago, IL, USA) was used for statistical analyses.

\section{Results}

The median follow-up of the participants was $6 \cdot 1$ years. One hundred and fifty-five participants initially free of MetS were diagnosed as incident cases of this syndrome (sixty women and ninety-five men). The overall cumulative incidence of MetS in this population was $2.9 \%$ during the follow-up period $(1 \cdot 7 \%$ in women and $5 \cdot 1 \%$ in men, $P<0 \cdot 001$ ).

The characteristics of the participants according to MetS status and sex are shown in Table 1. In both sexes, participants with MetS were older and had higher BMI at baseline. Among participants with MetS, the incidence of each MetS component was as follows: abdominal obesity, $100.0 \%$ in women and $100.0 \%$ in men (this is a sine qua non according to the IDF definition); high blood pressure, $73.3 \%$ in women and $72.6 \%$ in men; hyperglycaemia, $61.7 \%$ in women and $63.2 \%$ in men; hypertriacylglycerolaemia, $45 \cdot 0 \%$ in women and $46.5 \%$ in men; and low HDL-C, $38.3 \%$ in women and $33.7 \%$ in men. With respect to MetS components incidence according to sex in the whole population, abdominal obesity, hypertriacylglycerolaemia, hyperglycaemia and hypertension 
Table 1 Characteristics of the study participants according to MetS status, stratified by sex: the SUN (Seguimiento Universidad de Navarra, University of Navarra Follow-up) Project

\begin{tabular}{|c|c|c|c|c|c|c|c|}
\hline & \multicolumn{4}{|c|}{ MetS } & & & \multirow[b]{3}{*}{$P$} \\
\hline & \multicolumn{2}{|c|}{ Yes } & \multicolumn{2}{|c|}{ No } & \multicolumn{2}{|c|}{ Total } & \\
\hline & Mean or $n$ & SD or $\%$ & Mean or $n$ & SD or $\%$ & Mean or $n$ & SD or $\%$ & \\
\hline \multicolumn{8}{|l|}{ FEMALE $(n$ 3451) } \\
\hline Age (years) (mean, sD) & $44 \cdot 6$ & $10 \cdot 7$ & $33 \cdot 0$ & $9 \cdot 0$ & $33 \cdot 2$ & $9 \cdot 2$ & $<0.001 \dagger$ \\
\hline BMI $\left(\mathrm{kg} / \mathrm{m}^{2}\right)(\text { mean, } \mathrm{sD})^{*}$ & $24 \cdot 0$ & $2 \cdot 4$ & $21 \cdot 4$ & $2 \cdot 4$ & $21 \cdot 4$ & $2 \cdot 3$ & $<0.001+$ \\
\hline \multicolumn{8}{|l|}{ MetS components $(n, \%)$} \\
\hline Abdominal obesity* & 60 & $100 \cdot 0$ & 1329 & $39 \cdot 2$ & 1389 & $40 \cdot 2$ & $<0.001 \ddagger$ \\
\hline Hypertriacylglycerolaemia* & 27 & $45 \cdot 0$ & 53 & $1 \cdot 6$ & 80 & $2 \cdot 3$ & $<0.001 \ddagger$ \\
\hline Low HDL-C & 23 & $38 \cdot 3$ & 154 & $4 \cdot 5$ & 177 & $5 \cdot 1$ & $<0.001 \ddagger$ \\
\hline Hyperglycaemia* & 37 & $61 \cdot 7$ & 143 & $4 \cdot 2$ & 180 & $5 \cdot 2$ & $<0.001 \ddagger$ \\
\hline Hypertension* & 44 & $73 \cdot 3$ & 281 & $8 \cdot 3$ & 325 & $9 \cdot \overline{4}$ & $<0.001 \neq$ \\
\hline \multicolumn{8}{|l|}{ MALE ( $n$ 1866) } \\
\hline Age (years) (mean, sD) & $45 \cdot 6$ & $12 \cdot 0$ & $37 \cdot 3$ & $10 \cdot 7$ & $37 \cdot 7$ & $10 \cdot 9$ & $<0.001 \dagger$ \\
\hline BMI $\left(\mathrm{kg} / \mathrm{m}^{2}\right)(\text { mean, } \mathrm{sD})^{*}$ & $25 \cdot 7$ & $2 \cdot 0$ & $24 \cdot 2$ & $2 \cdot 2$ & $24 \cdot 3$ & $2 \cdot 2$ & $<0.001 \dagger$ \\
\hline \multicolumn{8}{|l|}{ MetS components $(n, \%)$} \\
\hline Abdominal obesity* & 95 & $100 \cdot 0$ & 793 & $44 \cdot 8$ & 888 & $47 \cdot 6$ & $<0.001 \ddagger$ \\
\hline Hypertriacylglycerolaemia* & 44 & $46 \cdot 3$ & 57 & $3 \cdot 2$ & 101 & $5 \cdot 4$ & $<0.001 \ddagger$ \\
\hline Low HDL-C & 32 & $33 \cdot 7$ & 67 & $3 \cdot 8$ & 99 & $5 \cdot 3$ & $<0.001 \ddagger$ \\
\hline Hyperglycaemia* & 60 & $63 \cdot 2$ & 146 & $8 \cdot 2$ & 206 & $24 \cdot 0$ & $<0.001 \ddagger$ \\
\hline Hypertension* & 69 & $72 \cdot 6$ & 379 & $21 \cdot 4$ & 448 & $24 \cdot 0$ & $<0.001 \ddagger$ \\
\hline
\end{tabular}

MetS, metabolic syndrome; HDL-C, HDL cholesterol.

MetS components: abdominal obesity, waist circumference $\geq 80 \mathrm{~cm}$ in women or $\geq 90 \mathrm{~cm}$ in men or BMl $\geq 30 \mathrm{~kg} / \mathrm{m}^{2} ; \mathrm{hypertriacylglycerolaemia,} \mathrm{TAG} \geq 150 \mathrm{mg} / \mathrm{dl}$; low HDL-C, HDL-C $<50 \mathrm{mg} / \mathrm{dl}$ in women or $<40 \mathrm{mg} / \mathrm{dl}$ in men; hyperglycaemia, glucose $\geq 100 \mathrm{mg} / \mathrm{dl}$; hypertension, blood pressure $\geq 130 / 85 \mathrm{mmHg}$.

${ }^{\star} P<0.001$ (Pearson's $\chi^{2}$ test) for comparison of incidence of MetS components between sexes.

$+P$ from ANOVA.

$\ddagger P$ from Pearson's $\chi^{2}$ test.

were more frequent among men than among women $(P<0 \cdot 001)$. These associations remained statistically significant after adjusting for age (data not shown).

The main characteristics of the participants according to category of body shape at age 5 years and sex are presented in Table 2. In both sexes, participants who classified themselves as being in the lowest body shape category (F1/M1) were older and had a higher incidence of MetS. On the other hand, participants who classified themselves as being in the highest body shape category (F6/M6 to F9/M9) were mostly men. In both sexes, the participants in this group also had higher average BMI at baseline.

The association between childhood body weight (categorical variable) and the incidence of adult MetS, stratified by sex, is shown in Table 3. Among men, both childhood underweight $(\mathrm{OR}=5 \cdot 20,95 \% \mathrm{CI} 1 \cdot 87,14 \cdot 50)$ and childhood obesity $(\mathrm{OR}=4 \cdot 66,95 \% \mathrm{CI} 1 \cdot 40,15 \cdot 51)$ were associated with adult MetS after adjusting for age and BMI at baseline. No association was apparent among women.

The associations between childhood body weight (continuous variable), weight gain during adolescence/ young adulthood (continuous variable) and incidence of adult MetS, stratified by sex, are presented in Table 4 . Among men, body shape at age 5 years was inversely related to adult MetS after adjusting for age and BMI at baseline (OR $=0.83,95 \%$ CI $0.72,0.97)$. This estimate can be interpreted as follows: for each 1-unit increase in body shape at age 5 years, the likelihood of adult MetS decreased by $17 \%$. On the other hand, weight gain from 5 to 20 years of age was directly associated with adult MetS after adjusting for age and BMI at baseline $(\mathrm{OR}=1 \cdot 49,95 \%$ CI $1 \cdot 01,2 \cdot 18$; Table 4$)$, meaning that for each 1-unit increase in body shape from 5 years to 20 years, the likelihood of adult MetS increased by $49 \%$. No association was apparent among women.

\section{Discussion}

Only among men, but not among women, we found that both childhood underweight and childhood obesity were associated with an increased risk of adult MetS; body shape at age 5 years was inversely and monotonically related to adult MetS. However, this association was reversed when we assessed change in body shape from 5 to 20 years. Weight gain during this period (childhood to adolescence/young adulthood) was associated with a higher risk of adult MetS.

Our findings are partially consistent with the results of a similar cohort study developed with 91453 adult French women who were asked to report their body silhouette at various ages, although the outcome was not MetS but diabetes ${ }^{(19)}$. Nevertheless, MetS can be considered a prestage of diabetes ${ }^{(1)}$. In that French study, body silhouettes at 8 years, at menarche and in young adulthood (20 to 25 years) were inversely associated with the risk of diabetes, independently of adult BMI during follow-up. In midadulthood ( 35 to 40 years) the association was reversed, 


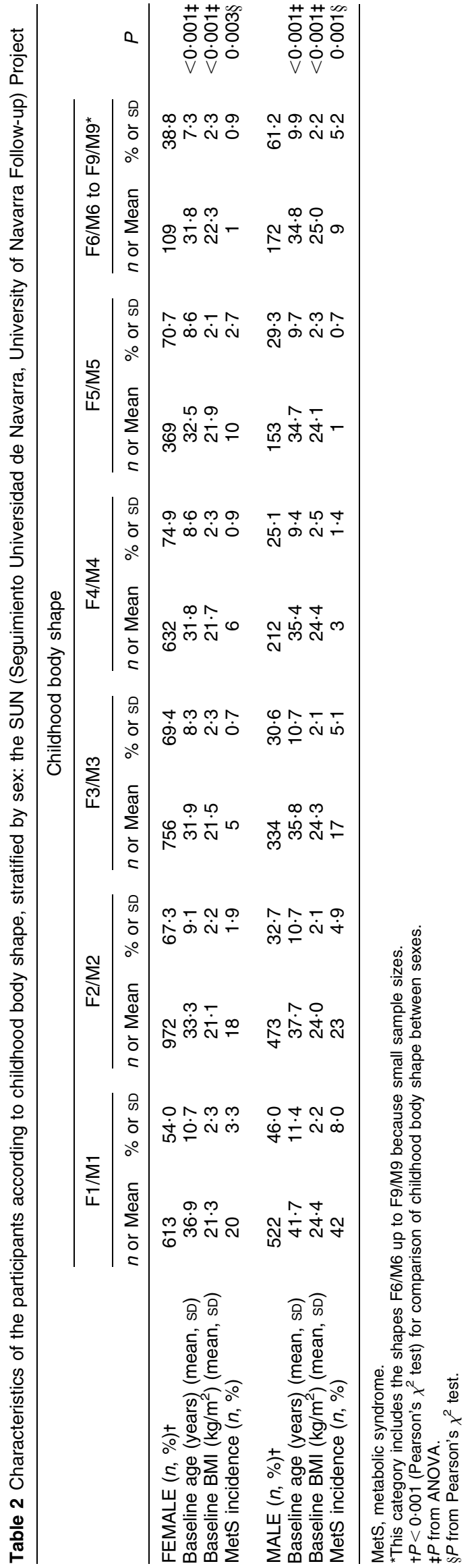

with an increase in risk related to a large body silhouette. Furthermore, an increase in body silhouette from childhood to mild-adulthood amplified the risk of diabetes ${ }^{(19)}$.

Three articles based on a cohort of 1492 men and women aged 26 to 32 years in Delhi (India) investigated the associations between patterns of infant, childhood and adolescent BMI and the incidence of chronic diseases in adulthood. Unlike our work and that done in France, BMI was calculated with directly measured weight and height. Despite the methodological dissimilarity, their results were in accordance with our findings. In one of these Indian studies, both lower BMI in infancy and rapid childhood BMI gain (2 to 11 years) were associated with an increased risk of adult MetS ${ }^{(20)}$. The results of the second study showed that the growth of children in whom impaired glucose tolerance or diabetes subsequently developed was characterized by low BMI between birth and 2 years of age, a young age at adiposity rebound (as defined by the age after infancy at which BMI starts to rise) and a sustained accelerated gain in BMI until adulthood $^{(21)}$. The last Indian investigation presented important results for understanding which are the critical periods of early life when weight gain is likely to have differential effects on the acquisition of fat or lean adult mass and on the development of chronic disorders ${ }^{(11)}$. Lower BMI at age 2 years was associated with earlier adiposity rebound, and this last condition predicted an increase in all measurements of adult body composition except height. Furthermore, BMI gains during infancy and early childhood were more strongly correlated with adult lean residual mass than with adult adiposity or with central adiposity. Greater BMI gains in late childhood (approximately between the ages of 6 and 7 years) and adolescence were strongly associated with adult adiposity and central adiposity. Other works have shown that this last relationship increased the risk of adult hypertension $^{(22)}$, type 2 diabetes $^{(23)}$ and IHD $^{(24)}$.

A likely mechanism to explain the associations between low BMI in infancy, accelerated BMI gain in later childhood and adolescence, and adult chronic diseases includes a low adult muscle mass as a key element. Skeletal muscle cells lose their ability to divide in early postnatal life. Therefore, as has been demonstrated in animal models, undernutrition at this period of life permanently diminishes muscle mass whereas improved nutrition at later ages produces an excessive fat deposition ${ }^{(25)}$. Sachdev et al. proposed that infancy, like fetal life, is a critical phase for lean mass growth. So, the susceptibility of some persons to chronic diseases may partly be caused by poor development of lean mass in fetal life and infancy, combined with increased adiposity in later childhood ${ }^{(11)}$.

With respect to MetS aetiology, excess body fat, especially visceral fat, mobilizes NEFA in the circulation. Afterwards, the rise of NEFA and their metabolites in insulin-sensitive tissues and cells, such as the hepatic and the muscular, promotes degradation in glucose capture 
Table 3 Association between childhood body weight and incidence of adult MetS, stratified by sex: the SUN (Seguimiento Universidad de Navarra, University of Navarra Follow-up) Project

\begin{tabular}{|c|c|c|c|}
\hline & \multicolumn{3}{|c|}{ Childhood body weight } \\
\hline & $\begin{array}{c}\text { Underweight } \\
\text { (F1/M1 to } \mathrm{F} 3 / \mathrm{M} 3)\end{array}$ & $\begin{array}{c}\text { Normal weight } \\
\text { (F4/M4 and F5/M5) }\end{array}$ & $\begin{array}{l}\text { Overweight/obesity } \\
\text { (F6/M6 to F9/M9) }\end{array}$ \\
\hline \multicolumn{4}{|l|}{ FEMALE } \\
\hline Cases & 43 & 16 & 1 \\
\hline Total & 2341 & 1001 & 109 \\
\hline MetS incidence/100 & $1 \cdot 8$ & $1 \cdot 6$ & 0.9 \\
\hline $95 \% \mathrm{Cl}$ & $1 \cdot 3,2 \cdot 5$ & $0 \cdot 9,2 \cdot 6$ & $0 \cdot 0,5 \cdot 0$ \\
\hline \multicolumn{4}{|l|}{ Model } \\
\hline OR & 0.95 & 1.00 & 0.57 \\
\hline $95 \% \mathrm{Cl}$ & $0 \cdot 52,1 \cdot 75$ & Ref. & $0 \cdot 73,4 \cdot 42$ \\
\hline \multicolumn{4}{|l|}{ MALE } \\
\hline Cases & 82 & 4 & 9 \\
\hline Total & 1329 & 365 & 172 \\
\hline MetS incidence/100 & $6 \cdot 2$ & $1 \cdot 1$ & $5 \cdot 2$ \\
\hline $95 \% \mathrm{Cl}$ & $5 \cdot 0,7 \cdot 6$ & $0 \cdot 4,3 \cdot 0$ & $2 \cdot 4,9 \cdot 7$ \\
\hline \multicolumn{4}{|l|}{ Model } \\
\hline OR & $5 \cdot 20$ & 1.00 & $4 \cdot 66$ \\
\hline $95 \% \mathrm{Cl}$ & $1 \cdot 87,14 \cdot 50$ & Ref. & $1 \cdot 40,15 \cdot 51$ \\
\hline
\end{tabular}

MetS, metabolic syndrome; Ref., reference category.

Model: OR and $95 \% \mathrm{Cl}$ are adjusted for age and $\mathrm{BMI}$ at baseline.

Table 4 Association between childhood body weight, weight gain during childhood to adolescence/young adulthood and incidence of adult metabolic syndrome, stratified by sex: the SUN (Seguimiento Universidad de Navarra, University of Navarra Follow-up) Project

\begin{tabular}{lccc}
\hline & OR & $95 \% \mathrm{Cl}$ & $P$ \\
\hline FEMALE & & & \\
$\quad$ Childhood body weight (continuous) & 0.94 & $0.77,1.14$ & 0.508 \\
$\quad$ Weight gain (continuous) & 0.97 & $0.50,1.87$ & 0.921 \\
MALE & & & \\
$\quad$ Childhood body weight (continuous) & 0.83 & $0.72,0.97$ & 0.019 \\
$\quad$ Weight gain (continuous) & 1.49 & $1.01,2 \cdot 18$ & 0.043 \\
\hline
\end{tabular}

$\mathrm{OR}$ and $95 \% \mathrm{Cl}$ are adjusted for age and $\mathrm{BMI}$ at baseline.

and insulin resistance. Moreover, the NEFA overflow to the liver stimulating glyconeogenesis, TAG production, VLDL cholesterol secretion and decreases insulin hepatic clearance $^{(1,8)}$

In the presence of hypertriacylglycerolaemia, a diminution of the cholesterol content and an addition of TAG occur in HDL-C, making this lipoprotein small and dense. These alterations cause an increased HDL-C renal clearance, diminishing its levels in plasma ${ }^{(1,8)}$.

As a product of the increased plasma glucose levels, there is a higher insulin release by the pancreas. This hyperinsulinaemia is associated with hypertension in the MetS aetiology because the insulin surplus leads to elevated renal sodium re-absorption, inducing a plasmatic volume expansion and, consequently, increasing the blood pressure. Furthermore, sympathetic nervous system activity is augmented in the hyperinsulinaemic state, promoting a higher adrenaline release. This neurohormone also increases the sodium re-absorption, the peripheral vasoconstriction, the heart rate, the adipose tissue lipolysis and the hepatic glyconeogenesis ${ }^{(1,8)}$.
It could be contradictory to think of childhood underweight as a public health problem in Spain, given that childhood obesity has high rates in Western developed countries. However, a study that investigated the trends in excess weight and thinness among Spanish schoolchildren in the period 1992-2004 demonstrated that both problems have increased concurrently. The overall prevalence of overweight/obesity increased from $24.4 \%$ in 1992 to $30 \cdot 9 \%$ in $2004(P=0 \cdot 07)$, and the overall prevalence of thinness was $2 \cdot 7 \%$ in 1992 and $9 \cdot 2 \%$ in 2004 $(P<0 \cdot 001)^{(26)}$. The authors speculate that the increase in thinness might be due to earlier sexual maturation, greater participation in sports and keener concern about controlling body weight in children.

We also found a significant association between childhood obesity and adult MetS in men $(\mathrm{OR}=4.66$, $95 \%$ CI $1 \cdot 40,15 \cdot 51$ ) after adjusting for age and BMI at baseline, which corroborates findings in other studies $^{(27,28)}$. It has been shown that overweight and obese children and adolescents are more likely to become obese adults ${ }^{(28)}$. Excess body fat, especially visceral fat, is a fundamental component of $\operatorname{Met} S^{(1,8)}$.

Regarding the absence of association for the female sex, this could be explained because of the lower percentage of women who were diagnosed with MetS during follow-up. Therefore, our cohort might be underpowered to assess these associations among women.

Our study has some limitations. Childhood and adolescence/young adulthood body weight was not directly measured but self-reported. The assessment was based on recalled body shape of the participants at ages 5 and 20 years.

Moreover, 69\% of our study participants classified themselves as underweight at age 5 years. In respect of 
this fact, we have to add a cautionary comment because a 30-year recollection of body shape as a surrogate for BMI is likely to be associated with a sizeable degree of measurement error. However, the validity of the remote recall of body shape has been demonstrated and the potential bias would be more likely towards the null value ${ }^{(14)}$. The MetS components were also not measured directly. However, these self-reported components by participants of the SUN Project were validated in specific study ${ }^{(18)}$. Furthermore, $>45 \%$ of our participants were health professionals. As a consequence, their self-reported information is likely to be of better quality than that other studies based on self-report ${ }^{(29)}$.

An additional limitation in our study is related to possible unmeasured confounders, since we adjusted only for age and adult BMI. The lack of control for potential confounders also invites caution in the interpretation of our findings. However, adjustment for potential important known confounders did not substantially change the observations from the crude analysis; and even those changes were away from the null. Therefore, we do not consider residual confounding as the most likely explanation for our results.

Some strengths of the present study are its prospective design, the inclusion of a high number of participants, and a long-term follow-up enabling us to assume a sufficiently large induction period and to avoid reverse causation.

\section{Conclusions}

In summary, childhood underweight and weight gain during childhood to adolescence/young adulthood increased the chance of adult MetS development among men. These results, if causal and confirmed in other prospective studies, support treating childhood underweight and weight gain during childhood to adolescence/young adulthood among men as part of comprehensive adult MetS prevention efforts.

\section{Acknowledgements}

The SUN Project has received funding from the Spanish Ministry of Health (grants PI030678, PI040233, PI070240, PI070312, PI081943, PI1002293, PI1002658, RD06/0045 and G03/140), the Navarra Regional Government (PI141/ 2005 and PI36/2008) and the University of Navarra. The authors declare no conflict of interest. A.M.P. carried out the statistical analysis, drafted the manuscript and gave final approval of the version to be published; J.J.B. made substantial contributions to analysis and interpretation of the data, was involved in drafting the manuscript, revised it critically for important intellectual content and gave final approval of the version to be published; A.S.-V. was involved in drafting the manuscript, revised it critically for important intellectual content and gave final approval of the version to be published; M.B-R. made substantial contributions to analysis and interpretation of the data, was involved in drafting the manuscript, revised it critically for important intellectual content and gave final approval of the version to be published; M.A.M.-G. made substantial contributions to conception and design of the study, acquisition, analysis and interpretation of the data; was involved in drafting the manuscript, revised it critically for important intellectual content and gave final approval of the version to be published. We thank all members of the SUN Study Group for administrative, technical and material support. We thank the SUN Project participants for their continued cooperation and participation.

\section{References}

1. Cornier MA, Dabelea D, Hernandez TL et al. (2008) The metabolic syndrome. Endocr Rev 29, 777-822.

2. Ford ES (2004) The metabolic syndrome and mortality from cardiovascular disease and all-causes: findings from the National Health and Nutrition Examination Survey II Mortality Study. Atherosclerosis 173, 309-314.

3. Salaroli LB, Barbosa GC, Mill JG et al. (2007) Prevalence of metabolic syndrome in population-based study, Vitória, ES-Brazil. Arq Bras Endocrinol Metab 51, 1143-1152.

4. Buckland GG, Salas-Salvadó J, Serra-Majem L et al. (2009) Increase in metabolic syndrome as defined by ATPIII from 1992-1993 to 2002-2003 in a Mediterranean population. Nutr Rev 67, Suppl. 1, S117-S125.

5. Lawes $\mathrm{CM}$, Vander Hoorn $\mathrm{S} \&$ \& Rodgers $\mathrm{A}$; International Society of Hypertension (2008) Global burden of bloodpressure-related disease, 2001. Lancet 371, 1513-1518.

6. Wild S, Roglic G, Green A et al. (2004) Global prevalence of diabetes: estimates for the year 2000 and projections for 2030. Diabetes Care 27, 1047-1053.

7. Regitz-Zagrosek V, Lehmkuhl E \& Mahmoodzadeh S (2007) Gender aspects of the role of the metabolic syndrome as a risk factor for cardiovascular disease. Gend Med $\mathbf{4}$, Suppl. B, S162-S177.

8. Eckel RH, Grundy SM \& Zimmet PZ (2005) The metabolic syndrome. Lancet 365, 1415-1428.

9. Silveira VM \& Horta BL (2008) Birth weight and metabolic syndrome in adults: meta-analysis. Rev Saude Publica $\mathbf{4 2}$, 10-18.

10. Vanhala M (1999) Childhood weight and metabolic syndrome in adults. Ann Med 31, 236-239.

11. Sachdev HS, Fall CH, Osmond C et al. (2005) Anthropometric indicators of body composition in young adults: relation to size at birth and serial measurements of body mass index in childhood in the New Delhi birth cohort. Am J Clin Nutr 82, 456-466.

12. Martínez-González MA, Sanchez-Villegas A, De Irala J et al. (2002) Mediterranean diet and stroke: objectives and design of the SUN project. Seguimiento Universidad de Navarra. Nutr Neurosci 5, 65-73.

13. Stunkard A (2000) Old and new scales for assessment of body image. Percept Mot Skills 90, 930.

14. Must A, Willett WC \& Dietz WH (1993) Remote recall of childhood height, weight, and body build by elderly subjects. Am J Epidemiol 138, 56-64.

15. Bes-Rastrollo M, Pérez JR, Sánchez-Villegas A et al. (2005) Validación del peso e índice de masa corporal auto-declarados de los participantes de una cohorte de graduados universitarios. Rev Esp Obes 3, 352-358. 
16. Alberti KG, Zimmet P \& Shaw J (2006) Metabolic syndrome: a new worldwide definition. A consensus statement from the International Diabetes Federation. Diabet Med 23, 469-480.

17. Klein S, Allison DB, Heymsfield SB et al. (2007) Waist circumference and cardiometabolic risk: a consensus statement from Shaping America's Health: Association for Weight Management and Obesity Prevention; NAASO; The Obesity Society; the American Society for Nutrition; and American Diabetes Association. Am J Clin Nutr 85, 1197-1202.

18. Fernandez-Montero A, Bes-Rastrollo M, Barrio MT et al. (2010) Validación de los componentes del síndrome metabólico. Nutr Hosp 25, Suppl. 1, 62.

19. de Lauzon-Guillain B, Balkau B, Charles MA et al. (2009) Birth weight, body silhouette over the life course and incident diabetes in 91,453 middle-aged women from the French E3N cohort. Diabetes Care 33, 298-303.

20. Fall CH, Sachdev HS, Osmond C et al. (2008) Adult metabolic syndrome and impaired glucose tolerance are associated with different patterns of BMI gain during infancy: data from the New Delhi Birth Cohort. Diabetes Care 31, 2349-2356.

21. Bhargava SK, Sachdev HS, Fall CH et al. (2004) Relation of serial changes in childhood body-mass index to impaired glucose tolerance in young adulthood. $N$ Engl J Med 350, $865-875$.
22. Law CM, Shiell AW, Newsome CA et al. (2002) Fetal, infant, and childhood growth and adult blood pressure: a longitudinal study from birth to 22 years of age. Circulation 105, 1088-1092.

23. Eriksson JG, Forsen T, Tuomilehto J et al. (2003) Early adiposity rebound in childhood and risk of type 2 diabetes in adult life. Diabetologia 46, 190-194.

24. Eriksson JG, Forsen T, Tuomilehto J et al. (2001) Early growth and coronary heart disease in later life: longitudinal study. BMJ 322, 949-953.

25. McCange RA (1962) Food, growth, and time. Lancet 2, 671-676.

26. Martínez-Vizcaíno V, Sánchez López M, Moya Martínez P et al. (2009) Trends in excess weight and thinness among Spanish schoolchildren in the period 1992-2004: the Cuenca study. Public Health Nutr 12, 1015-1018.

27. Srinivasan SR, Myers L \& Berenson GS (2002) Predictability of childhood adiposity and insulin for developing insulin resistance syndrome (syndrome $\mathrm{X}$ ) in young adulthood: the Bogalusa Heart Study. Diabetes 51, 204-209.

28. Allcock DM, Gardner MJ \& Sowers JR (2009) Relation between childhood obesity and adult cardiovascular risk. Int J Pediatr Endocrinol 2009: 108187.

29. Alonso A, Segui-Gomez M, de Irala J et al. (2006) Predictors of follow-up and assessment of selection bias from dropouts using inverse probability weighting in a cohort of university graduates. Eur J Epidemiol 21, 351-358. 